\title{
A Sensor Interface for Mobile Phones
}

\author{
Jens Rabe and Manfred Rost \\ Martin-Luther-Universität Halle-Wittenberg \\ Institute of Physics, 06099 Halle, Germany
}

\section{Introduction and motivation}

- Why a sensor interface for mobile phones?

A mobile phone seems to be an ideal instrument for monitoring vital parameters of patients or old people 11/. For this purpose an interface is needed to connect different sensors like photo sensors, accleration sensors or biosensors to the mobile phone in a simple way.

Furthermore, a mobile phone is also helpful for other sensor-based applications, e.g. in environmental monitoring and measuring technics.

- Is a mobile phone applicable for sensor measurements?

Mobile phones, especially smartphones, have all components of a typical hand held meter (display, input elements, microcontroller, memory etc.) without the typical sensor interface. Modern smartphones' processing power is comparable to PCs of the Pentium-2 class, and they have some helpful interfaces like Bluetooth, Wi-Fi or USB. A further important point is that mobile phones are widely used by virtually everyone.

- Are there already similar solutions?

A lot of special technical solutions using a mobile phone to get vital data are described in patent applications (see e.g. /2, 3/)

\section{Idea and realization of an interface}

The task is to design a universal sensor interface for mobile phones from different manufacturers.

Our sensor interface solution /4/ includes the interface hardware (sensor modules, measurement module, communication module e.g. USB or Bluetooth) and two software components:

- a Java ME program running on the mobile phone and

- $\quad$ an embedded software running on the microcontroller inside the interface.

Both software components act together in a master-slave-mode (Fig. 1).

The Java software allows to configure the interface and start a measuring process. Furthermore, it includes procedures to receive and visualize the measured values in an adequate manner (Fig. 2).

The embedded software controls the measuring process and the communication with the mobile phone via USB or Bluetooth.

\section{Prototype and first experiences}

With our interface prototype and different sensor adapters we are able to measure different physical or chemical parameters under control of a mobile phone. The communication to this phone is carried out wire-based (USB) or optionally wireless (Bluetooth).

Currently, prototypes of adapters with different complexities have been realized: Simpler ones to measure voltages or currents and more complex ones for $\mathrm{pH}$ values, finger pulse, temperature or acceleration.

A prototype of our interface with the $\mathrm{pH}$ sensor is shown in Fig. 3 . In this example USB is used to realize the connection to the "M600i" mobile phone by Sony-Ericsson.

The $\mathrm{pH}$ sensor needs a special amplifier. This amplifier is built into the connector (which is a phono jack); it guarantees a high input impedance and an amplification factor of 3. 
The software contains components to calibrate a new system or readjust previously calibrated values. For these tasks we use $\mathrm{pH}$ calibration solutions and a calibration function. The flow chart in Fig. 4 illustrates the calibration cycle and the co-action between the sensor, interface and mobile phone for a $\mathrm{pH}$ calibration.

Timing of the data transfer (Fig. 5, 6)

Since the MobileMeter software is based on Java ME and Java is an interpreted language, the execution performance is slower than with a compiled program. Especially rich animated graphics are only possible to some simple point yet. Moreover, it depends on the implementation of the Java Virtual Machine on the phone how concurrently running threads are treated.

With our lab phones, the Sony-Ericsson M600i, we managed to read up to approximately 250 values per second and display them in a scrolling $\mathrm{x} / \mathrm{t}$ diagram without running into buffer overflows.

The Bluetooth module we use on our interface hardware communicates with the microcontroller either with 9600 or 115200 baud. Using 9600 baud it is theoretically possible to transfer 1200 bytes per second, but because of attenuation and a changing geometry of the RF link, data transmission errors might occur. Because of these errors and slowdowns which result from instruction processing times in the microcontroller, a stable data rate of 800 to 1000 bytes per second if possible. The values to be graphically displayed are scaled so every value fits into a single byte. This makes it possible to measure and transfer 800 to 1000 values per second with equidistant time steps. So, when using Bluetooth, the maximum measuring rate is limited by the phone.

With USB the transfer rate between the communication module and the microcontroller is fixed at 9600 baud. But since all data transfers are carried out via "intermediate files", there are other delays. Establishing a USB connection and opening the file for writing takes 3 seconds. Then, as described above, 1000 values can be written to the file in one second. Another second is taken closing the file and the USB connection. Reading the 1000 values on the phone takes the sixth second. These considerations limit the number of values which can be transferred in one second to about 150 .

The values are measured in equidistant time steps and buffered in the microcontroller. After a software configurable time, the transfer of the contents of the buffer is initiated. Taking into consideration the timing of these processes limits the number of values to an even smaller number of approximately 50 per second (Fig. 6)

\section{Problems}

During the development we found some special smartphone-related problems, e.g.:

- mobile phone manufacturers restrict the possibilities of external software to protect the system from attacks (Malware, viruses, etc.);

- external software has to be signed (we use a VeriSign certificate for Sony-Ericsson UIQ based Phones /5/);

- mobile phone interfaces like the "Fast Port" or "Pop Port" don't have voltage outputs to power an external system.

\section{Literature}

11/ VDE-Positionspapier: Telemonitoring zur Prävention von Diabetes-Erkrankungen (www.vde-mikromedizin.de)

/2/ T. Simon, H.E. Urban:

Offenlegungsschrift DE 10219675 A1 (2002)

/3/ G. Flügel, L. Knüpfer, H. Kröger, M. Stoll:

Offenlegungsschrift DE 102005006024 A1 (2005)

14/ M. Rost, J. Rabe: Patent submitted

/5/ www.verisign.com 

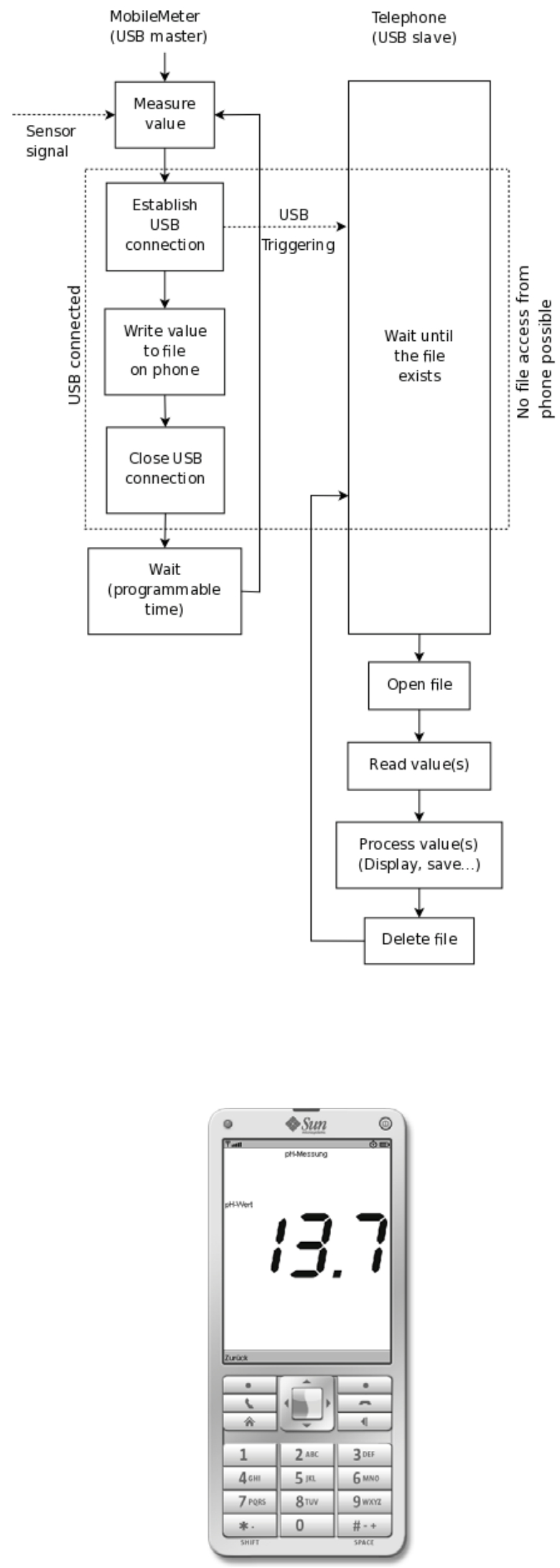

Fig. 1:

Master slave workflow for measuring process using USB (data handling similar in a dual port RAM)
Fig. 2:

Visualization of measuring values using very large seven-segment characters (software designed) 


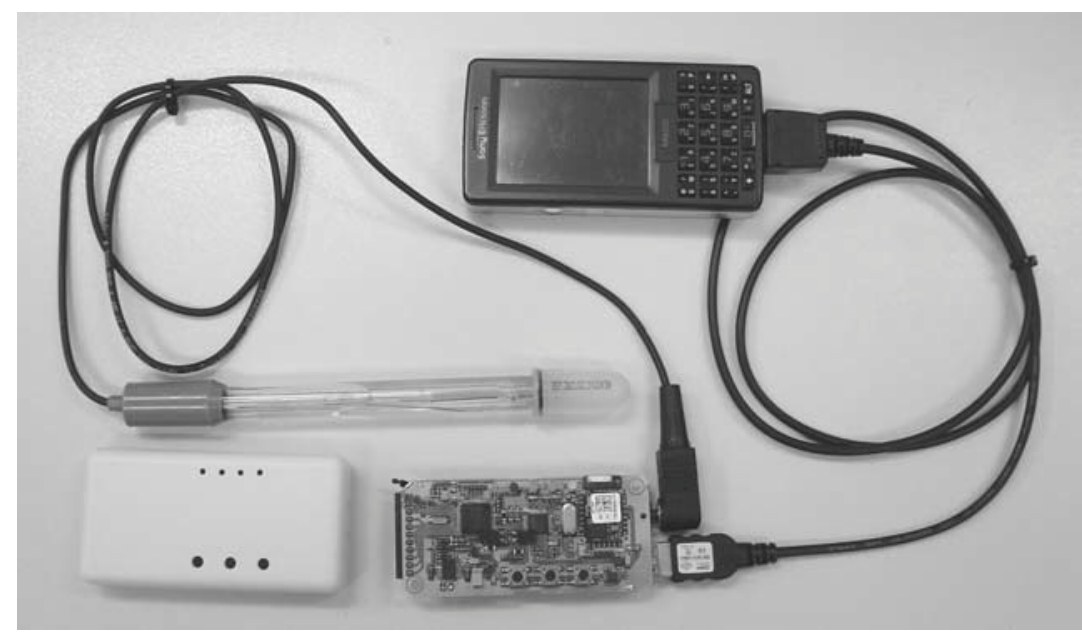

Fig.3:

Prototype of interface (outside case) with $\mathrm{pH}$ sensor and mobile phone

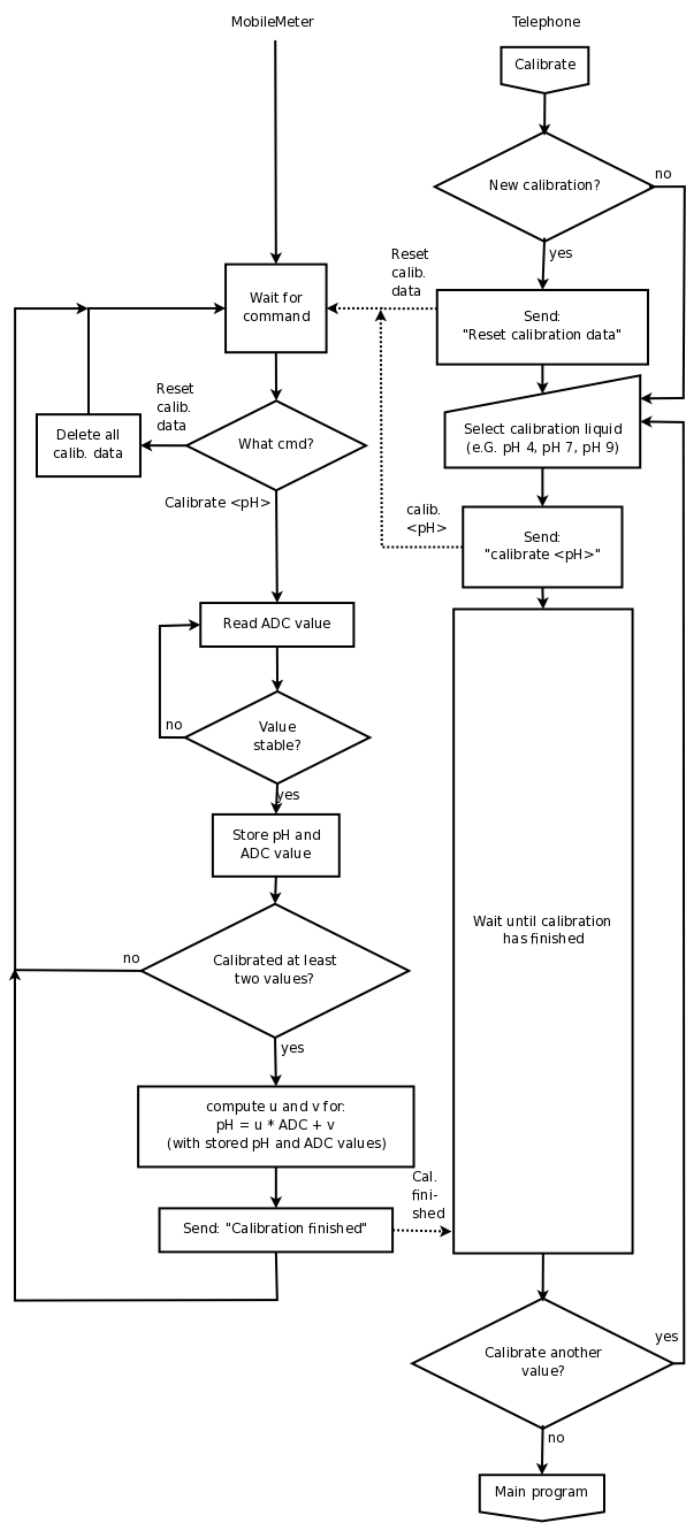

Fig. 4:

Flow chart of $\mathrm{pH}$ calibration cycle using the interface and a mobile phone 


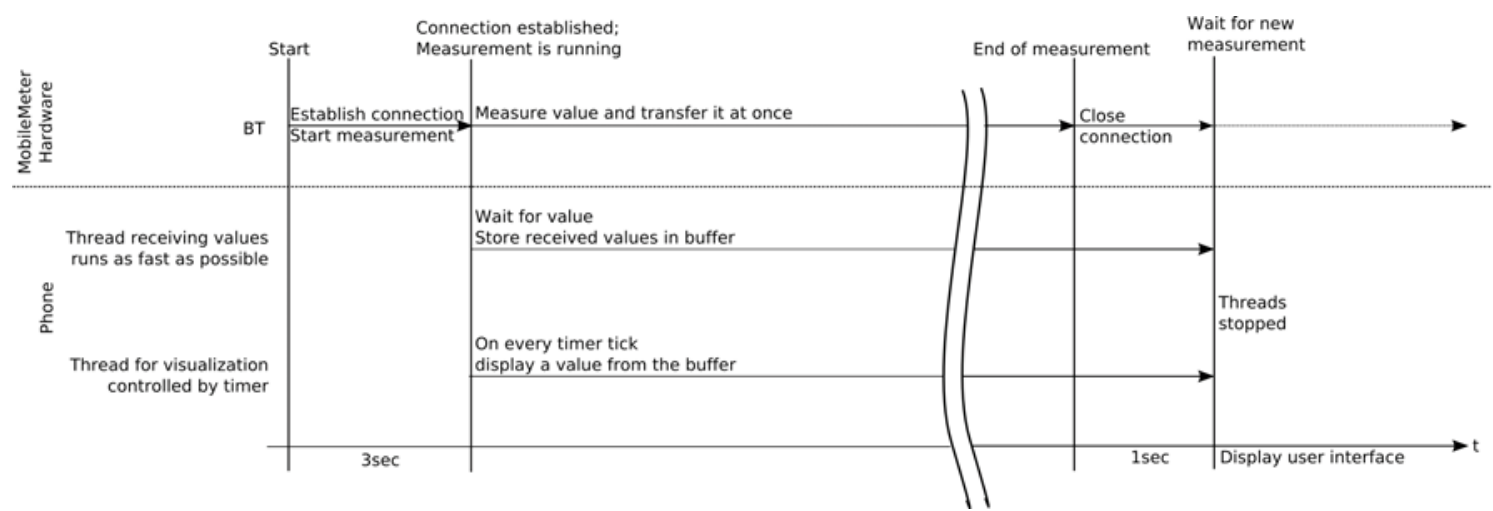

Fig. 5: Timing of the data transfer using Bluetooth

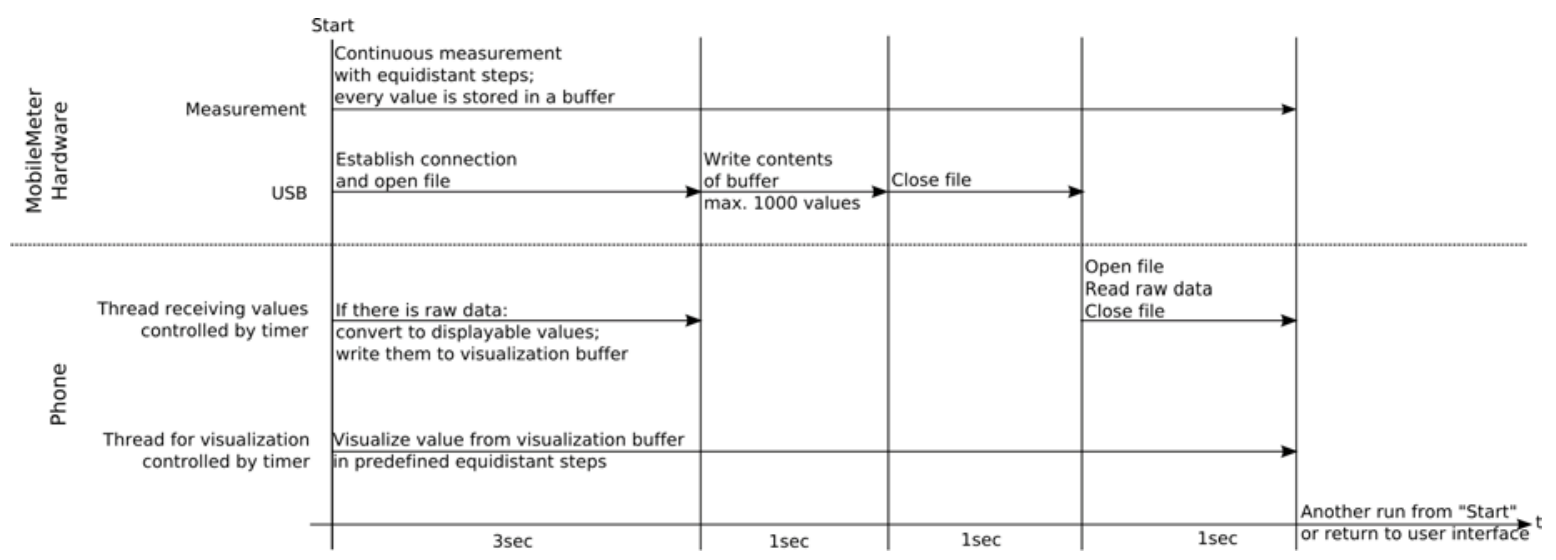

Fig. 6: Timing of the data transfer using USB 